\title{
Statically vs dynamically balanced gait: analysis of a robotic exoskeleton compared with a human
}

\author{
Giulia Barbareschi, Rosie Richards, Matt Thornton, Tom Carlson. IEEE Member, Catherine \\ Holloway, IEEE Member.
}

\begin{abstract}
In recent years exoskeletons able to replicate human gait have begun to attract growing popularity for both assistive and rehabilitative purposes. Although wearable robots often need the use of external support in order to maintain stability, the REX exoskeleton by REX Bionics is able to selfbalance through the whole cycle. However this statically balanced gait presents important differences with the dynamically balanced gait of human subjects. This paper will examine kinematic and kinetic differences between the gait analysis performed on a subject wearing the REX exoskeleton and human gait analysis data as presented in literature. We will also provide an insight on the impact that these differences can have for both rehabilitative and assistive applications.
\end{abstract}

\section{INTRODUCTION}

Millions of people around the world suffer from mobility impairments; in 2012 it was estimated that around 6.5 million people in the UK alone live with reduced mobility [1].

In recent years the combination of technological advances and dramatic increase in the ageing population has generated a growing interest in the development of assistive robotic devices. Wearable lower limbs exoskeletons have currently found applications within both rehabilitative and assistive technologies Depending on their purpose and application exoskeletons can be used with or without additional support such as crutches or an external frame [2]. This choice will have a large effect on the stability of the system, influencing gait characteristics, since the ability to maintain balance is crucially important for human walking. The necessary mechanisms adopted to achieve stability during human gait are extremely complex and therefore difficult to simplify through biomechanical modelling. Nevertheless several models have been adopted though the years with the aim of studying and simulating human gait. The most widely used model to reproduce human gait in robotic applications is the Zero Moment Point (ZMP) [3]. The ZMP, exclusive to legged robotics, was proposed by Vukubratovic and Juricic in 1969 and is defined as "the point where the influence of

G. Barbareschi is with University College London, Department of Civil Environmental and Geomatic Engineering, London, United Kingdom (email: giulia.barbareschi.14@ucl.ac.uk)

R. Richards . was with Royal National Orthopaedic Hospital NHS Trust Brockley Hill,Stanmore, Middlesex, HA7 4LP. She is now at the VU University Medical Centre, De Boelelaan 1118, 1081 HZ Amsterdam (email r.richards@vumc.nl)

M.Thornton is with Royal National Orthopaedic Hospital NHS Trust Brockley Hill,Stanmore, Middlesex, HA7 4LP. (email: matt.thornton@rnoh.nhs.uk)

T. Carlson is with the ASPIRE CREATe Centre for Rehabilitation Engineering and Assistive Technology, University College London (email: t.carlson@ucl.ac.uk).

C.Holloway is with University College London, Department of Civil Environmental and Geomatic Engineering, London, United Kingdom (email:c.holloway@ucl.ac.uk) all forces acting on the mechanism can be replaced by one single force". For a system to be considered balanced the ZMP has to lie within the foot support area [4]

The human gait is defined as a dynamically balanced process, which includes statically unbalanced phases throughout the cycle. On the other hand, robotic gait often needs to be statically balanced during the whole cycle [5] When considering an exoskeleton the absence of an external support will imply that the exoskeleton must be able to maintain static stability through the whole cycle, guaranteeing the safety of the wearer at whole times, including in the event of power failure. This will consequentially reduce gait speed and step length [6]. If conditions related to the ZMP are satisfied for both statically and dynamically balanced systems there is an additional requirement in order to achieve static balance [5]. Studies performed by McGhee \& Frank [7] established that a gait pattern can be considered statically stable if the vertical projection of the Centre of Mass (COM) lies within the area of the supporting polygon. This research was mainly aimed at identifying requirements for statically balanced quadruped gait, however the rule can be considered valid also for biped gait. Although many studies have been carried out to design control systems able to achieve static and dynamic balance for humanoid legged robots, the implications of these two different modalities on kinematic and kinetic aspects have often been overlooked. To this end gait analysis was performed on an exoskeletal device during fixed pace treadmill walking and results where compared to standard human kinetics and kinematics parameters as they are presented in literature.

\section{DESIGN OF THE STUDY}

\section{A. Equipment}

The REX exoskeleton by REX Bionics was chosen as device for the testing. This was due to the fact that the Rex is the only completely self-supporting (i.e. no need for crutches) exoskeleton available among both the research and commercial prototypes. The device features five Degrees of Freedom (DoF) per leg, two at the hip, one at the knee and two at the ankle. Five brushed DC motors are employed as actuators for the respective DoFs [9]. The total weight of the device is $38 \mathrm{~kg}$ and the maximum load allowed for the user is $100 \mathrm{~kg}[10]$.

\section{A. Setting}

The pilot study was carried out in the Motor Learning Laboratory at the Royal National Orthopedic Hospital (RNOH) in Stanmore, Middlesex. The Laboratory is equipped with the Gait Real-time Analysis Interactive Lab (GRAIL) by Motek Medical featuring an instrumented double belt treadmill and ten Vicon opto-electric 


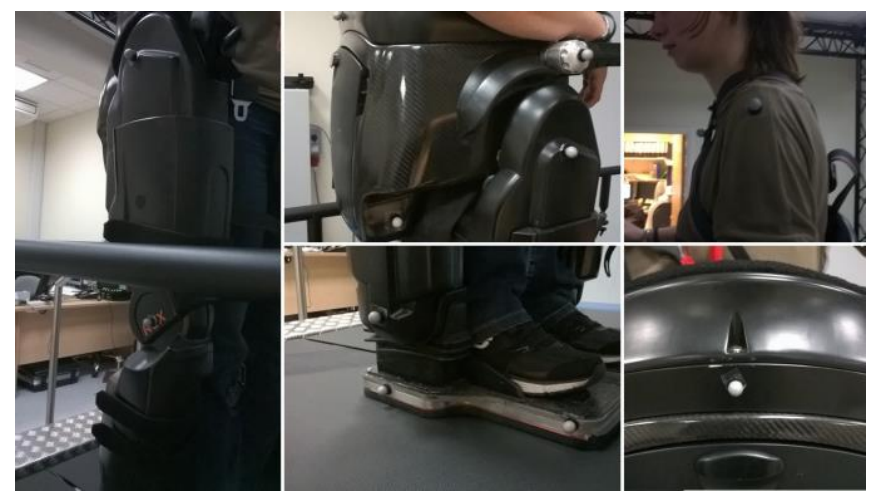

Figure 1 Position of the markers for gait analysis

cameras to record positions of retro-reflective markers within the capture volume.

Sampling frequency is $100 \mathrm{~Hz}$ for motion capture data and $1000 \mathrm{~Hz}$ for analog channels (i.e. force plate data) [8]. Due to the highly stereotyped gait exhibited by the exoskeleton and the early stages of the research, the analysis was performed on a single healthy subject (female, 28 years old, $62 \mathrm{~kg}$ ). No training was provided prior the experiment.

\section{Marker positioning}

In order to provide a complete three dimensional motion analysis, eighteen infrared reflecting passive markers were positioned in proximity of the exoskeleton joints and at strategic reference points on the user. Fig 1 shows the position of the markers for the experiment.

\section{Data COLLECTION AND PROCESSING}

The speed of the treadmill was set at $0.05 \mathrm{~m} / \mathrm{s}$, to accommodate the REX walking speed, the gradient was set at 0 degrees and the test was run for ten minutes. Analog and video data were collected for 62 seconds during steady state walking. This time corresponded to two strides of the exoskeleton. Marker displacement from motion capture data was obtained through the use of the C3D toolbox ${ }^{1}$ while analog data concerning Ground Reaction Forces (GRF) were extracted using the Biomechanical toolkit ${ }^{2}$ and a Matlab script was implemented to perform the analysis. The analysis was simplified to two-dimensional. This was considered sufficient since the movement of the exoskeleton is (primarily) in the sagittal plane. First it was necessary to compute the angular displacement of the foot, shank, and thigh in space using (1):

$$
\theta_{i j}=\arctan \frac{y_{j}-y_{i}}{x_{j}-x_{i}}
$$

Where $\mathrm{xj}$ and yj represent the coordinates on $\mathrm{x}$ and $\mathrm{y}$ axis of the proximal marker on the segment and xi and yi the coordinates along $\mathrm{x}$ and $\mathrm{y}$ axis of the distal marker on the segment.

Subsequently the absolute joint angles were calculated as (2):

$$
\theta_{\text {joint }}=\theta_{\text {proximal segment }}-\theta_{\text {distal segment }}
$$

Angular velocity $V_{x}$ and acceleration $A_{x}$ in each direction were determined using (3) and (4):

\footnotetext{
${ }^{1}$ https://www.c3d.org/appmatlab.html

${ }^{2} \mathrm{https}: / /$ code.google.com/p/b-tk/wiki/MatlabBinaries
}

$$
\begin{aligned}
V_{x} & =\frac{x_{i+1}-x_{i}}{\Delta t} \\
A_{x} & =\frac{v x_{i+1}-v x_{i}}{\Delta t}
\end{aligned}
$$

Where $x_{i}$ and $x_{i+1}$ represent two consecutive position of the marker in the $\mathrm{x}$ direction.

Graphs obtained after initial processing appeared noisy. This was likely due to the vibrations produced by the exoskeleton during gait combined with the high sampling frequency of the GRAIL system. Data were smoothed through application of a moving average filter with a window length of 100 samples.

Once kinematic values were calculated and the GRF extracted from analog channels it was necessary to establish anthropometric parameters for the exoskeleton. To this end the anthropometric table proposed by Kirtley based on Dempster and Jensen's original work was adopted to establish coordinates and values for COM and moment of inertia [11]. In order to calculate COM position and moment of inertia for each segment the following assumptions have been made:

- The total body mass of the combined REX-wearer system is $100 \mathrm{~kg}$ (38 kg Rex, $62 \mathrm{~kg}$ subject)

- The ratio between the segment mass and the total body mass for the combined REX-wearer system is assumed the same as the one between the segment and the human body

- The position of the markers on each segment determine their extremities. Thus the distance between them represent the length of the segment

Once the anthropometric parameters were estimated it was possible to apply inverse dynamics technique through the use of Euler equation (5):

$$
\sum M=I \alpha
$$

Joints reaction forces $R_{x}, R_{y}$ and moments were then calculated as shown by, [11] (6), (7), (8)

$$
\begin{gathered}
R_{x}=(m * a x)-F_{x} \\
R_{y}=(m * a y)-F_{y}+m g
\end{gathered}
$$

$$
M=F_{y}\left(x_{d}-x_{\text {COM }}\right)+F_{x}\left(y_{\text {COM }}-y_{d}\right)-R_{y}\left(x_{\text {COM }}-x_{p}\right)-
$$$$
R_{x}\left(y_{p}-y_{\text {COM }}\right)+I \alpha-M_{d}
$$

Where:

$m=$ the mass of the segment

$a_{x}$ and $a_{y}=$ linear acceleration of the COM in $\mathrm{x}$ and $\mathrm{y}$ direction

$F_{x}$ and $F_{y}=$ GRF in $\mathrm{x}$ and $\mathrm{y}$ direction

$g=$ gravitational acceleration

$x_{C O M}$ and $y_{C O M}=$ coordinates in $\mathrm{x}$ and $\mathrm{y}$ directions of the segment COM

$x_{d}$ and $y_{d}=$ coordinates in $\mathrm{x}$ and $\mathrm{y}$ directions of the distal marker on the segment

$x_{p}$ and $y_{p}=$ coordinates in $\mathrm{x}$ and $\mathrm{y}$ directions of the proximal marker on the segment

$I=$ moment of inertia of the segment

$\alpha=$ angular acceleration of the joint 
$M_{d}=$ moment of the joint caudal to the segment considered Finally the mechanical power was determined for each joint through (9)

$$
P=M \omega
$$

Where $\mathrm{M}$ is the joint moment and $\omega$ is the angular velocity.

\section{RESULTS AND DISCUSSION}

\section{A. Kinematics}

The data obtained through the process illustrated in the earlier paragraph were compared with human gait analysis presented by Winter, Fig 2 [12]

Initially the general pattern of hip and knee angular displacements do not present major differences between static and dynamic gait. Range of movement is generally smaller for robotic gait, which is consistent with the shorter step length of the REX. The hip joint appears to be always flexed through the whole cycle, causing a slight forward inclination of the wearer's upper body. This is likely due to the necessity to guarantee safety and comfort of the wearer by avoiding backwards trunk inclination which might lead to falls. The extension movement of the hip associated with the weight transfer between the backward and forward leg happens later and with a less steep curve for the static gait of the REX compared to human gait. Simultaneously the ankle exhibits an increasing dorsiflexion, in contrast to the human gait where toe off is characterized by plantar flexion. Although the curve pattern for the knee angular displacement looks similar between statically and dynamically balance gait, there is an important difference. The peak of maximum flexion during human walking, around $70 \%$ of the cycle, is normally associated with swing phase of the leg to guarantee foot clearance. During the static gait of the REX this peak seems to occur earlier and is considerably reduced compared to human walking. This occurs to allow the lowering of the body's Centre of Gravity (COG), which allows improved stability by maintaining the COM projection within the support polygon.
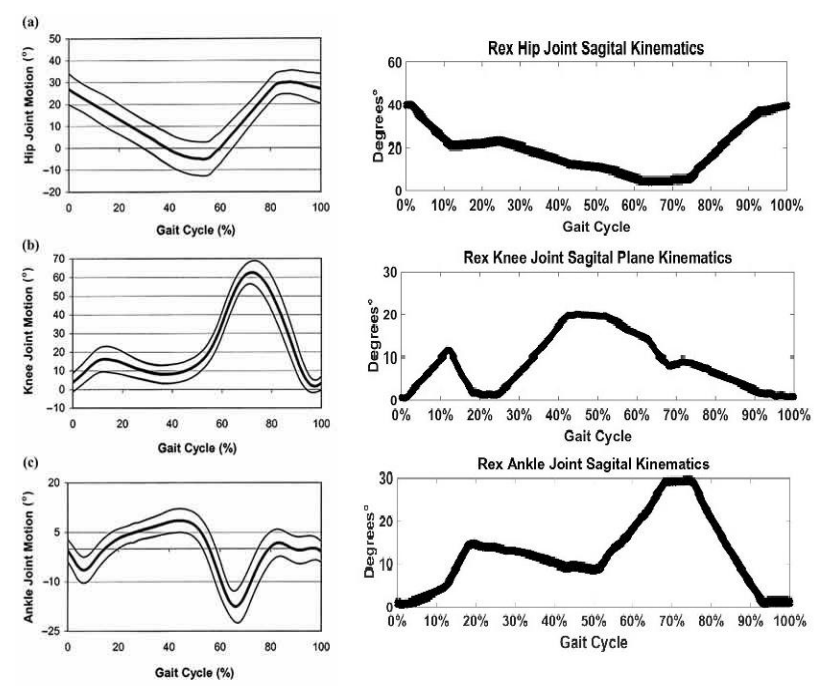

Figure 2 Angular displacements of joints during human [12] and robotic gait

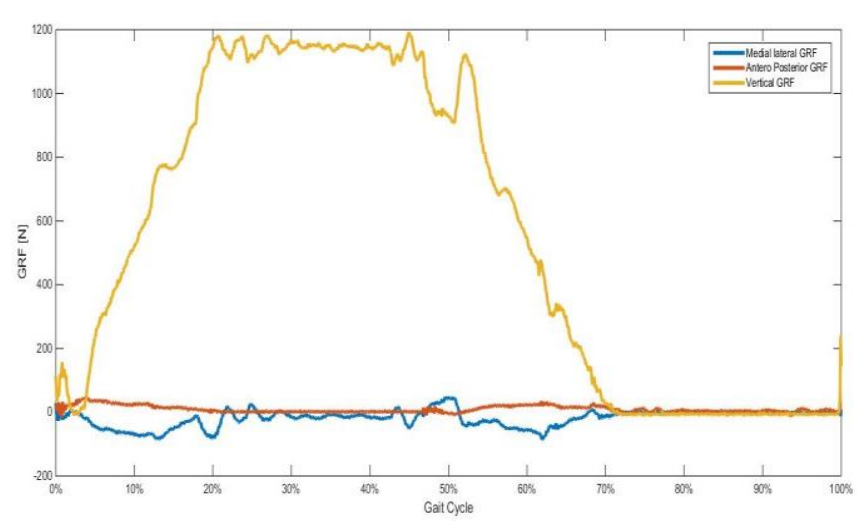

Figure 3 Ground Reaction Forces from REX gait analysis

\section{B. Kinetics}

The GRF associated with the static gait of the REX reveal some important characteristics Fig 3.

The slower translation of the body COM, due to the need for accurate control of its position, causes the almost complete elimination of antero-posterior GRF. For the same reason there is no reduction of the vertical component during double support phase, indicating that the COM projection is maintained within the area of the back foot until the whole body weight is transferred forward in preparation of swing phase. The vertical component shows higher values, with peaks reaching almost $120 \%$ of the total body weight. The medial-lateral GRF appears the most significant shear force with a peaks of $80 \mathrm{~N}$ between $10 \%$ and $20 \%$ of the cycle due to loading response and the translation of the COM within the support polygon. This is further confirmed when examining data related to the movement of the Centre of Pressure (CoP) along the antero-posterior and medial-lateral axis. The displacement of the $\mathrm{CoP}$ underneath the footplate during stance phase can be enclosed in a rectangular area of $16 \mathrm{~cm}$ width by $2 \mathrm{~cm}$ height, This suggest that continuous lateral adjustments are needed in order to maintain balance during stance phase.

The GRF's were used to calculate the moments acting at the ankle, knee and hip, which were compared to those of the classic human gait cycle presented by Winter [13], Fig 4 .

In the REX data is possible to notice the absence of dorsiflexor moment after heel strike because the whole surface of the footplate of the REX approaches the ground almost simultaneously, therefore negating the requirement for the first rocker. Additionally the peak plantarflexor value, characteristic of toe off, is not present since there is no active power generation at the ankle. By contrast to the human pattern, peak values for both ankle plantarflexor and knee extensor moment for the exoskeleton are maintained from $25 \%$ to $50 \%$ of the gait cycle indicating that the body COM remain stable during the prolonged single support phase. The extensor moment at the knee and plantarflexor moment at the ankle regulates the antigravitary response of the exoskeleton. When the weight begins to shift forward in preparation of the swing phase the hip begins to show a flexor moment which will continue until mid-swing phase when the leg will be gradually lowered to the ground. Joint powers during robotic gait, Fig 5, appear very different from what is expected during human gait. 


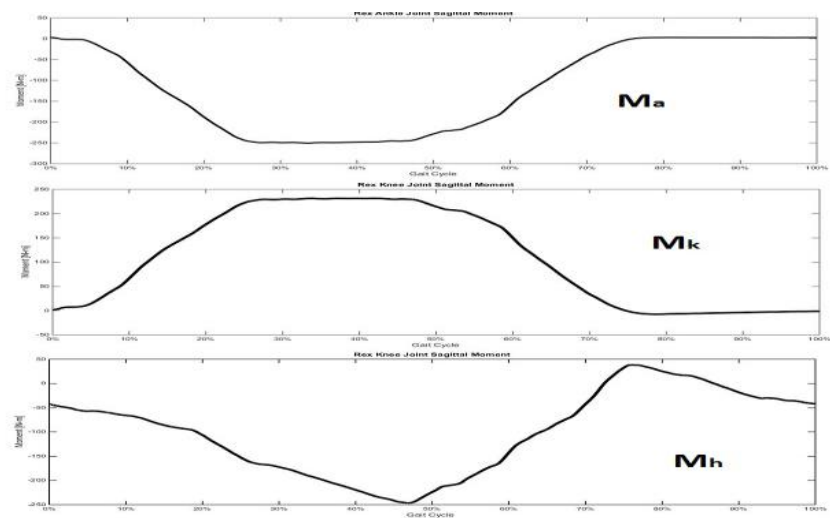

Figure 4 REX gait joint moments. From the top showing the moment at the ankle (Ma), the moment at the knee (Mk) and the moment at the hip (Mh)

At the ankle two region of negative power indicate power absorption necessary to regulate the position of the shank before and after single support phase. At the knee, there are two positive regions corresponding to concentric flexor activity needed to move the COM. A smaller one after loading response and a larger one during midstance when the knee flexion help maintaining the projection of the COM within the supporting polygon. The hip power is positive through almost the whole cycle. Two negative regions indicate eccentric activity to control hip flexion before single support phase and terminal swing. In contrast with the human gait, where the third rocket ankle plantar flexor generate most of the power for forward propulsion, the REX exhibits larger power generation at the knee and hip.

\section{CONCLUSION}

The numerous differences highlighted in this study, have shown the profound distinction between statically and dynamically balanced gait. For this reason an exoskeleton using a statically balanced gait, will likely find little application as a gait rehabilitation device, where the close replication of healthy human gait is the key goal. However, in terms of usage as an assistive technology, the ability to safely support the wearer through the whole cycle, while leaving the upper limbs free for additional tasks, can represent a great advantage. In this case, although the gait may not be natural, the risks of shoulder damage associated with crutch-use are also diminished. For patients where gait is not likely to be recovered, the preservation of upper limb functionality is arguably a higher priority than replicating a natural gait.

\section{FUTURE WORK}

Due to the stereotyped nature of the REX static gait it was chosen to perform the analysis on single subject. Additionally, a single right step was extracted during steady state walking and presented for kinematic and kinetic analysis. This was attributed to the subject opposing resistance to the exoskeleton movement. Analysis performed before and after training could, in the future confirm this hypothesis. Based on these preliminary results further testing has been planned to determine the importance of intra- and inter-subject variability.

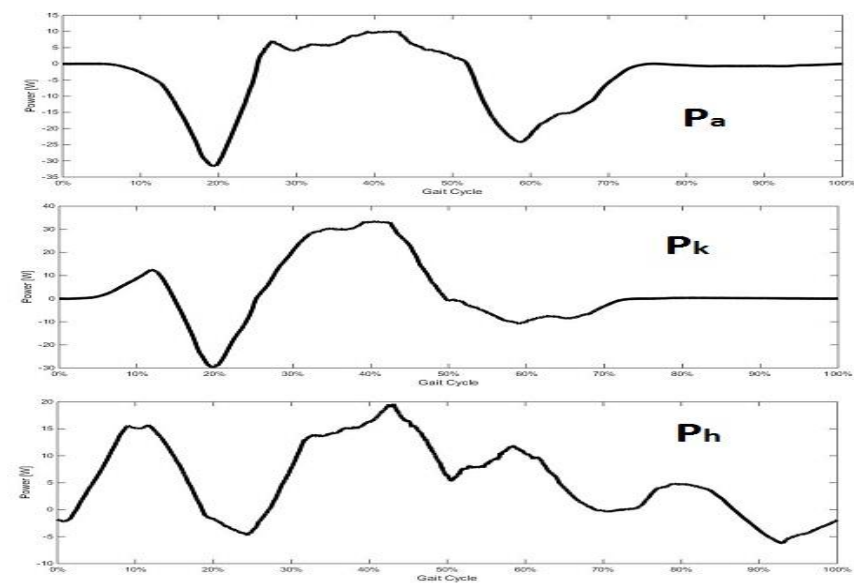

Figure 5 REX gait joint powers Top showing the power at the ankle (Pa), middle the power at the knee $(\mathrm{Pk})$ and bottom the power at the hip (Mh)

\section{ACKNOWLEDGMENTS}

This work was supported by EPSRC Program Grant "Transforming the Engineering of Cities". We are very grateful to REX Bionics for their support of the project.

\section{REFERENCES}

[1] Office for Disability Issues and Department for Work and Pensions, "Disability Prevalence estimates 2011/12" UK Government, 2014

[2] S. Mohammed, Y. Amirat, and H. Rifai, 'Lower-Limb Movement Assistance through Wearable Robots: State of the Art and Challenges', Advanced Robotics, vol. 26, no. 1-2, pp. 1-22, Jan. 2012.

[3] M. Vukobratović and B. Borovac, 'Zero-moment point - thirty five years of its life', Int. J. Human. Robot., vol. 01, no. 01, pp. 157-173, Mar. 2004.

[4] M. Vukobratovic and D. Juricic, 'Contribution to the Synthesis of Biped Gait', IEEE Transactions on Biomedical Engineering, vol. BME-16, no. 1, pp. 1-6, Jan. 1969.

[5] C. Mummolo, L. Mangialardi, and J. H. Kim, 'Quantifying Dynamic Characteristics of Human Walking for Comprehensive Gait Cycle', J Biomech Eng, vol. 135, no. 9, pp. 091006-091006, Jul. 2013.

[6] T. A. Swift, 'Control and Trajectory Generation of a Wearable Mobility Exoskeleton for Spinal Cord Injury Patients', eScholarship, Jan. 2011.

[7] R. B. McGhee and A. A. Frank, 'On the stability properties of quadruped creeping gaits’, Mathematical Biosciences, vol. 3, pp. 331351, Aug. 1968.

[8] Royal National Orthopaedic Hospital (2014, December 18). Motor Learning Laboratory. [Online] Available: https://www.rnoh.nhs.uk/clinical-services/rehabilitation-andtherapy/motor-learning-laboratory

[9] Shultz, A. (2012, January 31). Robotic exoskeleton: For a better quality of life. [Online] Available: http://www.maxonmotor.com/maxon/view/application/EXOSKELET $\mathrm{T}-\mathrm{AB}$

[10] Rex Bionics (2014, November 24). Rex Prescriber Guide. [Online] Available:http://webcache.googleusercontent.com/search?q=cache:M7 s6ikWtlJcJ:rexbionics.s3.amazonaws.com/wpcontent/uploads/2014/03/REX-Prescribers-Guide-V2.3-2014-0324. $\operatorname{doc} \mathrm{x}+\& \mathrm{~cd}=2 \& \mathrm{hl}=\mathrm{it} \& \mathrm{ct}=\mathrm{clnk} \& \mathrm{gl}=\mathrm{uk}$

[11] C. Kirtley, Clinical Gait Analysis: Theory and Practice. Elsevier Health Sciences, 2006, pp 120-124.

[12] D.A.Winter, The Biomechanics and Motor Control of Human Locomotion. 1987 p121

[13] D. A. Winter, Biomechanics and Motor Control of Human Movement John Wiley \& Sons, 2009, pp 104. 